\title{
SINS/BDS Tightly-Coupled Integrated Navigation Algorithm for Hypersonic Vehicle
}

kai chen ( $\nabla$ chenkai@nwpu.edu.cn )

Northwestern Polytechnical University

Sen-sen PEI

Northwestern Polytechnical University

Cheng-zhi ZENG

Northwestern Polytechnical University

Gang DING

China Airborne Missile Academy

\section{Research Article}

Keywords: Hypersonic vehicle, Strapdown inertial navigation algorithm, BeiDou Navigation Satellite System, Tightly-coupled integrated navigation

Posted Date: November 19th, 2021

DOI: https://doi.org/10.21203/rs.3.rs-1062630/v1

License: (1) (1) This work is licensed under a Creative Commons Attribution 4.0 International License. Read Full License 


\title{
SINS/BDS Tightly-Coupled Integrated Navigation Algorithm for Hypersonic Vehicle
}

\author{
Kai Chen ${ }^{1, *}$, Sen-sen PEI ${ }^{1}$, Cheng-zhi ZENG ${ }^{1}$, Gang DING ${ }^{2}$ \\ ${ }^{1}$ School of Astronautics, Northwestern Polytechnical University, Xi'an 710072, China \\ ${ }^{2}$ China Airborne Missile Academy, Luoyang 471009, China \\ *E-mail: chenkai@nwpu.edu.cn
}

\begin{abstract}
A tightly-coupled integrated navigation system (TCINS) for hypersonic vehicles is proposed when the satellite signals are disturbed. Firstly, the architecture of the integrated navigation system for the hypersonic vehicle is introduced. This system applies fiber SINS, BeiDou satellite receiver (BDS) and SOPC missile-born computer. Subsequently, the SINS mechanization for hypersonic vehicle is presented. The $\mathrm{J} 2$ model is employed for the normal gravity of the near space. An algorithm for updating the attitude, velocity and position is designed. State equations and measurement equations of SINS/BDS tightly-coupled integrated navigation for hypersonic vehicle are given, and a scheme of validity for satellite data is designed. Finally, the SINS/BDS tightly-coupled vehicle field tests and hardware-in-the-loop (HWIL) simulation tests are carried out. The vehicle field test and HWIL simulation results show that the heading angle error of tightly-coupled integrated navigation is within $0.2^{\circ}$, the pitch and roll angle errors are within $0.05^{\circ}$, the maximum velocity error is $0.3 \mathrm{~m} / \mathrm{s}$, and the maximum position error is $10 \mathrm{~m}$.
\end{abstract}

Keywords: Hypersonic vehicle; Strapdown inertial navigation algorithm; BeiDou Navigation Satellite System; Tightly-coupled integrated navigation

1. INTRODUCTION. Near space vehicles have advantages that vehicles such as traditional aircrafts and satellites do not have, and have great development potential, especially in terms of intelligence collection and long-range precision strike. Near space vehicles can be divided into low-dynamic and high-dynamic vehicles (Duan and Zhou, 2020). Hypersonic vehicle is the representative of the rapid development of high-dynamic vehicle in recent years (Niu et al., 2019), which adopts aerodynamic configuration design with high lift-drag ratio and propulsion technology such as hypersonic gliding, rocket engine or scramjet to achieve hypersonic flight (Chen et al., 2020a).

The navigation system is one of important parts in hypersonic vehicle, and is used to measure the position, velocity, and attitude of the vehicle. The strapdown inertial navigation system (SINS) has the advantages of full navigation information, high autonomy and high update rate. The GPS has global, all weather, constant and real-time navigation, positioning and timing functions (Schmidt, 2015). Due to the inherent complementary advantages of SINS and GNSS, the navigation schemes of hypersonic vehicles in various countries around the world are all mainly based on SINS and GNSS (Chen et al., 2019). X-43A hypersonic vehicles use Honeywell's mature H-764 series INS/GPS system (Bahm et al., 2005, Chen et al., 2020b). HTV-2 hypersonic vehicles apply a tightly-coupled GPS/IMU integrated navigation system with an accuracy of about 3 meters (Walker et al., 2008). X-51A hypersonic vehicle is equipped with inertial measurement unit (IMU) and GPS receiver (Hank et al., 2008). The navigation system of German SHEFEX-2 hypersonic vehicles 
integrates IMU, GPS receiver, and star sensor (STR), and uses IMU/GPS for integrated navigation (Steffes, 2012). Russian Avangard uses SINS/GLONASS/SAR integrated navigation scheme (Chen et al., 2020a). Hypersonic vehicle navigation systems in Japan and India also use SINS/GNSS integrated navigation systems.

The Chinese BeiDou Navigation Satellite System (BDS) (Fan et al., 2018) has global and all-daytime navigation, and positioning and timing capabilities, and unique short message communication services. BDS has been extensively applied in marine fisheries, transportation, meteorological forecasting, hydrological monitoring, geographic information of surveying and mapping, and communication unify-time (Ma et al., 2019). However, there are fewer application cases of BDS in hypersonic flight. In this paper, a SINS algorithm and a tightly-coupled integrated navigation algorithm are designed for the SINS/BDS integrated navigation system for hypersonic vehicle, the processing strategy of the navigation system is gived when the BeiDou satellite signal is disturbed, and vehicle field tests and hardware-in-the-loop simulation tests are also performed.

\section{SINS/BDS INTETRATED NAVIGATION SYSTEM HARDWARE}

2.1. Overall structure of SINS/BDS integrated navigation system. The SINS/BDS integrated navigation system is an important component of the flight control system in hypersonic vehicle. The flight control system includes a control system, a guidance system, and a navigation system. Flight control system hardware includes inertial measurement unit (IMU), BeiDou satellite receiver, actuator, seeker, information processing unit, peripheral interface, etc. (Figure 1).

2.2. Information processing unit. The SOPC technology is applied for the integrated design for the information processing unit. The SOPC chip integrates an ARM processor, a million-gate programmable logic array, and an IP core with multiple bus communication functions to complete the information exchange between the system and the external interface. The software runs on the real-time operating system of the information processing unit, and runs integrated navigation algorithms, control algorithms, and guidance algorithms, and flight timing and process management.

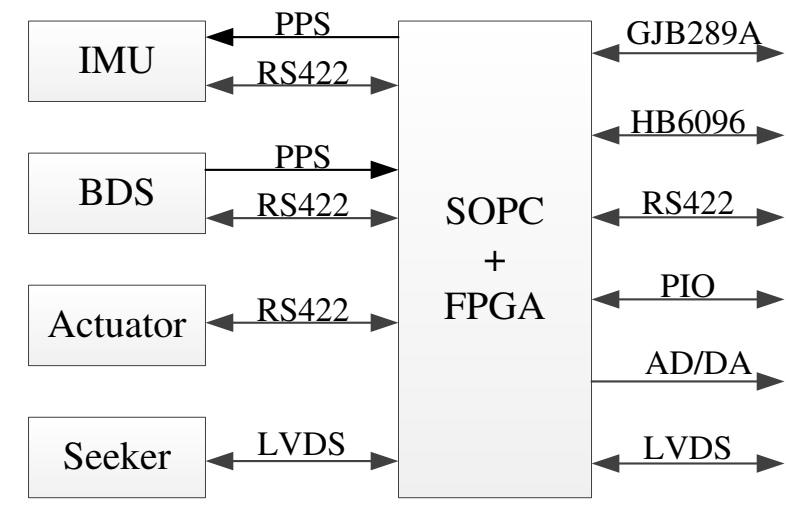

Figure 1. Composition of flight control components.

2.3. Inertial measurement unit. The inertial measurement unit (IMU) includes a three-axis fiber optic gyro cluster, a three-axis accelerometer cluster, an inertial measurement electronic cluster, and an inertial measurement secondary power supply (Figure 2). The inertial 
measurement electronic cluster includes amplifier circuit, temperature measurement circuit, and correction circuit.

The IMU measures the three-axis acceleration and angular velocity of a hypersonic vehicle, and has the characteristics of high dynamics, large range, high accuracy, and miniaturization, where the fiber optic gyro bias is $3^{\circ} / \mathrm{h}(1 \sigma)$ with $100 \mathrm{ppm}(1 \sigma)$ scale factor, and $0.5^{\circ} / \mathrm{h}(1 \sigma)$ white noise, and the gyro data output period of $2 \mathrm{~ms}$; and the accelerometer drift is $1 \times 10^{-3}$ $\mathrm{g}(1 \sigma)$ with the scale factor of $100 \mathrm{ppm}(1 \sigma)$, the white noise of $0.5 \times 10^{-4} \mathrm{~g}(1 \sigma)$, and the output period of $6 \mathrm{~ms}$.

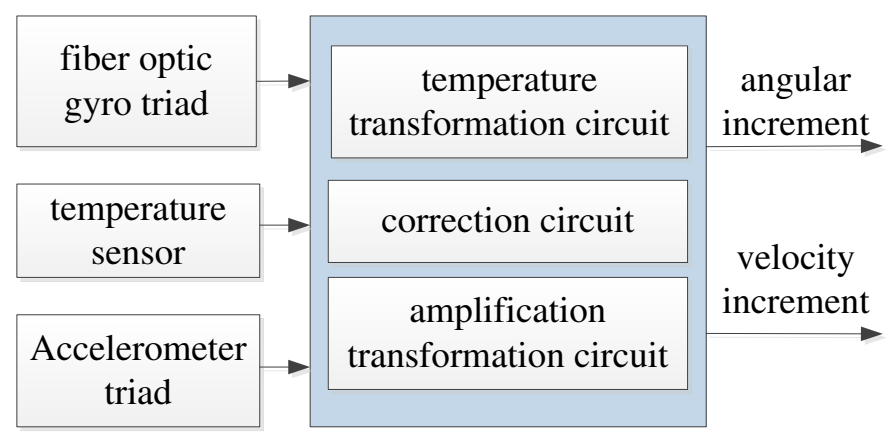

Figure 2. Functional principle diagram of the inertial measurement unit.

2.4. BDS satellite receiver. The BDS satellite receiver uses the "radio frequency $+\mathrm{ADC}+$ FPGA + DSP" framework for the integrated design. FPGA is used for acquisition and track of BDS satellite navigation signals, and DSP is used for satellite navigation positioning and logic processing (Figure 3). The receiver provides real-time velocity, position, and time information, as well as ephemeris, pseudo-range (PR), and pseudo-range rate (PRR) information for the hypersonic vehicle. At the same time, the positioning information is transmitted to the telemetry component for recording the flight trajectory of the hypersonic vehicle. The positioning accuracy of the satellite receiver is $10 \mathrm{~m}(1 \sigma)$, the velocity accuracy is $0.3 \mathrm{~m} / \mathrm{s}(1 \sigma)$, and the output period is $200 \mathrm{~ms}$.

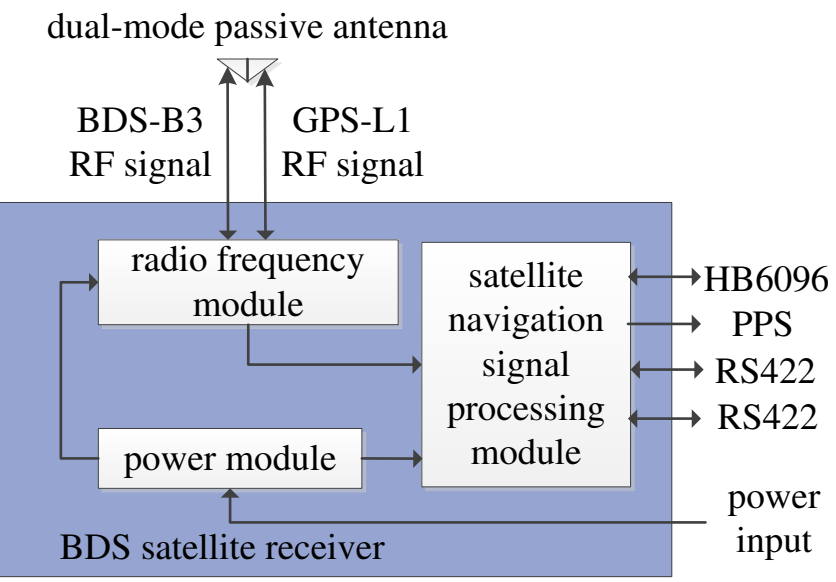

Figure 3. Diagram of the BDS satellite receiver.

\section{SINS/BDS TIGHTLY-COUPLED INTEGRATED NAVIGATION ALGORITHM}

3.1. Overall frame of the TCINS algorithm. The data flow diagram of the SINS/BDS TCINS 
algorithm is shown in Figure 4.

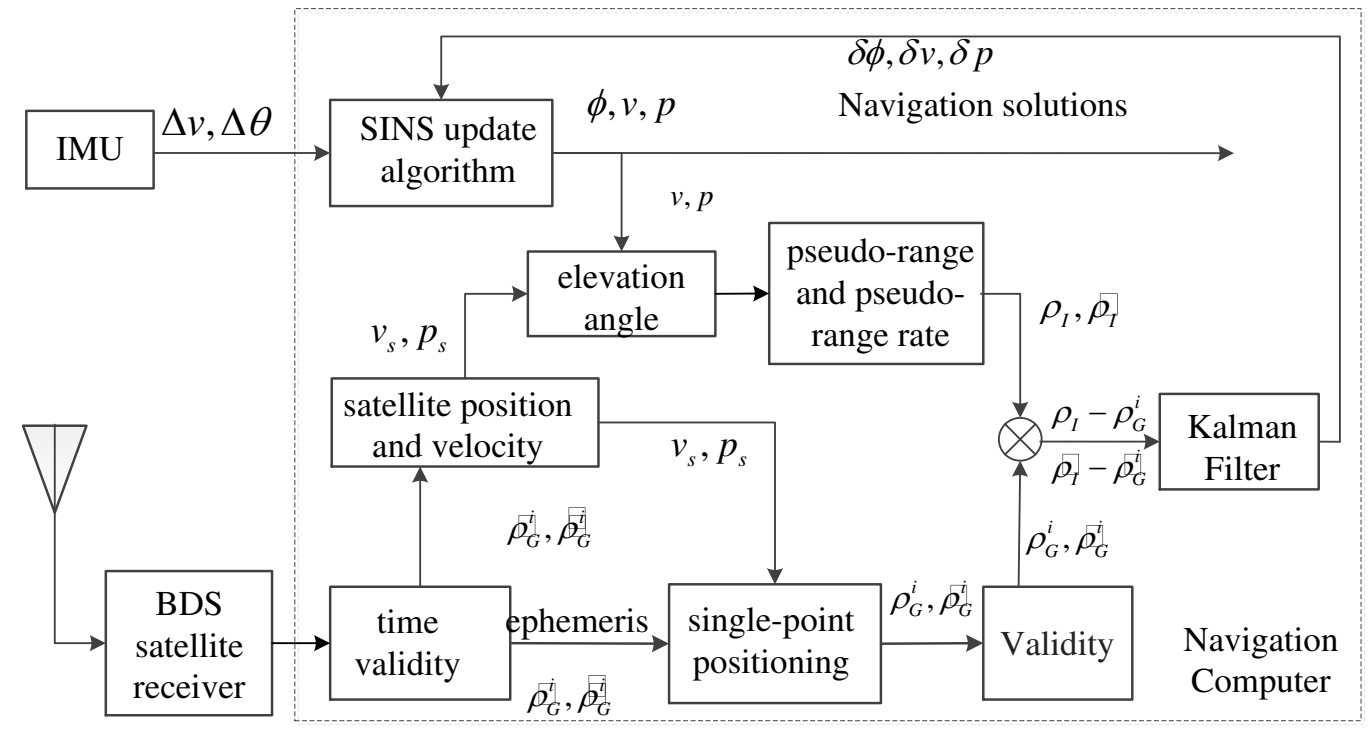

Figure 4. The data flow schematic diagram of the TCINS algorithm.

The implementation steps of the SINS/BDS TCINS algorithm are as follows.

(1) Periodically carry out numerical update for SINS algorithm according to the angular and velocity increments output by the IMU, and output the attitude $\phi$, velocity $v$, and position

$p$.

(2) When receiving the data of the BDS satellite receiver, determine the receiving time and accuracy factor. If the data is valid, continue to step (3), otherwise return to step (1).

(3) Perform incremental determination on the PR observations $\beta_{\xi}^{*}$ and PRR observations

\& of BDS receiver. If the data is valid, continue to step (4), otherwise return to step (1).

(4) Use the ephemeris data, $\beta_{g}^{*}$, and to calculate the position $p_{s}$ and velocity $v_{s}$ of the BDS receiver. Then calculate the satellite clock difference, ionospheric error, and tropospheric error based on the ephemeris data. Subsequently, perform single-point positioning to obtain the corrected $\rho_{G}^{i}$ and $\&_{G}^{i}$.

(5) Use $v, p$, and ephemeris to calculate the satellite elevation angle. Select the appropriate satellite according to the satellite elevation angle, and calculate $\rho_{I}$ and \& of SINS.

(6) The difference between $\rho_{I}$ and \& as well as the difference between $\rho_{G}^{i}$ and $\&_{G}^{i}$ are determined. If the determination is valid, they are combined through Kalman filtering, the error estimates $\delta \phi, \delta v$, and $\delta p$ are obtained, calculate SINS to output attitude $\phi$, 
velocity $v$, and position $p$, and perform correction. Otherwise return to step (1).

3.2. Mechanization of hypersonic vehicle SINS algorithm. The local-level (LL) frame is choiced as the reference frame for hypersonic vehicle. The strapdown inertial navigation equation is shown in equation (1) (Chen et al., 2018,).

$$
\left\{\begin{aligned}
\boldsymbol{Q} & =\frac{1}{2} \boldsymbol{q}_{b}^{n} \otimes \boldsymbol{\omega}_{n b}^{b} \\
\boldsymbol{k} \& & =\boldsymbol{R}_{b}^{n} \boldsymbol{f}^{b}-\left(2 \boldsymbol{\omega}_{i e}^{n}+\boldsymbol{\omega}_{e n}^{n}\right) \times \boldsymbol{v}^{n}+\boldsymbol{g}^{n} \\
\boldsymbol{P L}^{2} & =\boldsymbol{C}^{p} \boldsymbol{v}^{n}
\end{aligned}\right.
$$

where $\boldsymbol{q}^{n}$ is the attitude quaternion of the vehicle in the local-level frame; $\boldsymbol{\omega}_{n b}^{b}$ is the angular velocity; $\boldsymbol{v}^{n}$ is the velocity vector in LL frame; $\boldsymbol{f}^{b}$ is the specific force; $\boldsymbol{g}^{n}$ is the gravity vector of LL frame; $\boldsymbol{p}^{n}=\left[\begin{array}{lll}L & \lambda & H\end{array}\right]^{\mathrm{T}}$ is location.

Because the cruise altitude of the hypersonic vehicle is greater than the flight altitude of an aviation vehicle, this paper adopts the $\mathrm{J}_{2}$ gravity model in earth-centered earth-fixed (ECEF) frame (Chen et al., 2018, Chen et al., 2020b). The calculation method is shown in equation (2).

$$
\boldsymbol{g}^{n}=\boldsymbol{R}_{e}^{n} \boldsymbol{g}^{e}
$$

where $\boldsymbol{R}_{e}^{n}$ is the DCM of the ECEF frame to the LL frame, as shown in equation (3). The vector calculation of $\boldsymbol{g}^{e}=\left[g_{x}^{e}, g_{y}^{e}, g_{z}^{e}\right]^{\mathrm{T}}$ is shown in equation (4), and the calculation method is shown in equation (5).

$$
\begin{gathered}
\boldsymbol{R}_{e}^{n}=\boldsymbol{R}_{z}\left(90^{\circ}\right) \boldsymbol{R}_{y}\left(90^{\circ}-L\right) \boldsymbol{R}_{z}(\lambda) \\
\boldsymbol{g}^{e}=\boldsymbol{G}^{e}+\boldsymbol{\omega}_{i e} \times \boldsymbol{\omega}_{i e} \times \boldsymbol{r}^{e} \\
g_{x}^{e}=-\frac{x_{e} \mu}{r^{3}}\left[1+\frac{3 J_{2} a_{e}^{2}}{2 r^{2}}-\frac{15 J_{2} a_{e}^{2} z_{e}^{2}}{2 r^{4}}\right]+x_{e} \omega_{i e}^{2} \\
g_{y}^{e}=-\frac{y_{e} \mu}{r^{3}}\left[1+\frac{3 J_{2} a_{e}^{2}}{2 r^{2}}-\frac{15 J_{2} a_{e}^{2} z_{e}^{2}}{2 r^{4}}\right]+y_{e} \omega_{i e}^{2} \\
g_{z}^{e}=-\frac{z_{e} \mu}{r^{3}}\left[1+\frac{9 J_{2} a_{e}^{2}}{2 r^{2}}-\frac{15 J_{2} a_{e}^{2} z_{e}^{2}}{2 r^{4}}\right]
\end{gathered}
$$

In the equation (5), $\mu$ the Earth's gravitational coefficient, $J_{2}$ is the spherical harmonic coefficient of the Earth, $a_{e}$ is the equatorial radius of the Earth, and $r=\sqrt{x_{e}^{2}+y_{e}^{2}+z_{e}^{2}}$. $x_{e}, y_{e}, z_{e}$ is calculated based on the latitude and longitude $(L, \lambda, H)$ of the hypersonic vehicle, and calculated by the position $\boldsymbol{p}^{e}=\left[x_{e}, y_{e}, z_{e}\right]^{\mathrm{T}}$ in the ECEF frame, as shown in 
equation (6).

$$
\left\{\begin{array}{l}
x_{e}=(N+H) \cos L \cos \lambda \\
y_{e}=(N+H) \cos L \sin \lambda \\
z_{e}=\left[N\left(1-e^{2}\right)+H\right] \sin L
\end{array}\right.
$$

where $N=a_{e} / \sqrt{1-e^{2} \sin ^{2} L}$.

3.3. Strapdown inertial navigation numerical update algorithm. As mentioned above, the output periods of the gyro and the accelerometer are $2 \mathrm{~ms}$ and $6 \mathrm{~ms}$, respectively. The strapdown inertial navigation algorithm update period $T$ is designed to be $6 \mathrm{~ms}$. The gyroscope performs three equally interval samplings during the update cycle, and the angular increments are in order of $\boldsymbol{\Delta} \boldsymbol{\theta}_{1}, \boldsymbol{\Delta \boldsymbol { \theta } _ { 3 }}$, and $\boldsymbol{\Delta \boldsymbol { \theta } _ { 3 }}$. The accelerometer samples once during the update cycle, and the velocity increment is $\boldsymbol{\Delta} \boldsymbol{V}$. The designed strapdown inertial navigation numerical update algorithm is as follows (Savage, 1998a, 1998b).

3.3.1. Attitude update algorithm. According to the three samples of the gyroscope, the calculation method of rotation vector is

$$
\Phi_{m}=\Delta \theta_{1}+\Delta \theta_{2}+\Delta \theta_{3}+\frac{33}{80} \Delta \theta_{1} \times \Delta \theta_{3}+\frac{57}{80} \Delta \theta_{2} \times\left(\Delta \theta_{3}-\Delta \theta_{1}\right)
$$

Considering the rotation effect of the navigation coordinate system, the equivalent rotation vector $\boldsymbol{\eta}_{m}$ from $t_{m-1}$ to $t_{m}$ is

$$
\boldsymbol{\eta}_{m}=\boldsymbol{\Phi}_{m}-\boldsymbol{R}_{n(m-1)}^{b} \boldsymbol{\omega}_{i n(m-1)}^{n} T
$$

The quaternion $\boldsymbol{q}_{b(m)}^{b(m-1)}$ corresponding to $\boldsymbol{\eta}_{m}$ is

$$
\boldsymbol{q}_{b(m)}^{b(m-1)}=\cos \frac{\eta_{m}}{2}+\frac{\boldsymbol{\eta}_{m}}{\eta_{m}} \sin \frac{\eta_{m}}{2}
$$

Substitute the calculated $\boldsymbol{q}_{b(m)}^{b(m-1)}$ into equation (10) to complete the attitude update.

$$
\boldsymbol{q}_{b(m)}^{n}=\boldsymbol{q}_{b(m-1)}^{n} \boldsymbol{q}_{b(m)}^{b(m-1)}
$$

where $\boldsymbol{q}_{b(m-1)}^{n}$ is the attitude quaternion at $t_{m-1}$, and $\boldsymbol{q}_{b(m)}^{n}$ is the attitude quaternion at $t_{m}$.

3.3.2. Velocity update algorithm. From the specific force equation of equation (1), the velocity update algorithm can be obtained as follows.

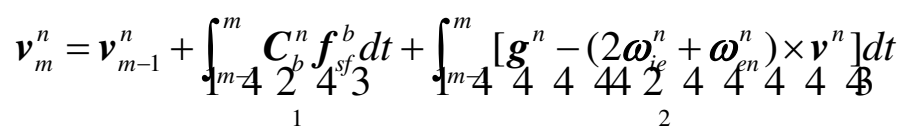

where the first term is caused by the velocity increment, which is $\Delta \boldsymbol{v}_{s f(m)}^{n}$; and the second 
term is caused by the acceleration term, which is $\Delta \boldsymbol{v}_{g / \operatorname{cor}(m)}^{n}$.

The calculation method for the first term is

$$
\Delta \boldsymbol{v}_{s f, m}^{n}=\boldsymbol{R}_{n(m-1)}^{n(m)} \boldsymbol{R}_{b(m-1)}^{n(m-1)} \boldsymbol{\Delta} \boldsymbol{V}
$$

where $\boldsymbol{R}_{n(m-1)}^{n(m)} \approx \boldsymbol{I}-\left(\boldsymbol{\varsigma}_{\frac{m}{2}} \times\right), \quad \boldsymbol{\varsigma}_{\frac{m}{2}}=\frac{1}{2} \omega_{i n(m-1)}^{n} T$

The calculation method for the second term is

$$
\Delta \boldsymbol{v}_{g / c o r(m)}^{n}=\left[\boldsymbol{g}_{m-1}^{n}-\left(2 \boldsymbol{\omega}_{i e(m-1)}^{n}+\boldsymbol{\omega}_{e n(m-1)}^{n}\right) \times \boldsymbol{v}_{m-1}^{n}\right] T
$$

Bring equations (12) and (13) into equation (11) to complete the velocity update.

3.3.3. Position update algorithm. The position update is as shown below (Chen et al., 2020b).

$$
\left\{\begin{array}{l}
L_{m}=L_{m-1}+\frac{v_{N, m-1}^{n}+v_{N, m}^{n}}{2\left(R_{M, m-1}+H_{m-1}\right)} T \\
\lambda_{m}=\lambda_{m-1}+\frac{v_{E, m-1}^{n}+v_{E, m}^{n}}{2\left(R_{N, m-1}+H_{m-1}\right) \cos L_{m-1}} T \\
H_{m}=H_{m-1}+\frac{v_{U, m-1}^{n}+v_{U, m}^{n}}{2} T
\end{array}\right.
$$

\subsection{SINS/BDS tightly-coupled filter.}

3.4.1. SINS/BDS tightly-coupled state equation. SINS/BDS tightly-coupled filter state vectors include attitude error $\phi$, velocity error $\delta \boldsymbol{v}^{n}$, position error $\delta \boldsymbol{p}$, gyroscope drift $\boldsymbol{\varepsilon}^{b}$, and accelerometer drift $\nabla^{b}$, and BDS equivalent clock error $\delta t_{u}$ and equivalent clock frequency error $\delta t_{r u}$.

The state equation of the SINS/BDS filter is as follows. The state transition matrix $\boldsymbol{F}(t)$ is described in reference (Gao et al., 2017; Dai et al., 2018; Han et al., 2018).

$$
\boldsymbol{X}^{\ell}(t)=\boldsymbol{F}(t) \boldsymbol{X}(t)+\boldsymbol{G}(t) \boldsymbol{W}(t)
$$

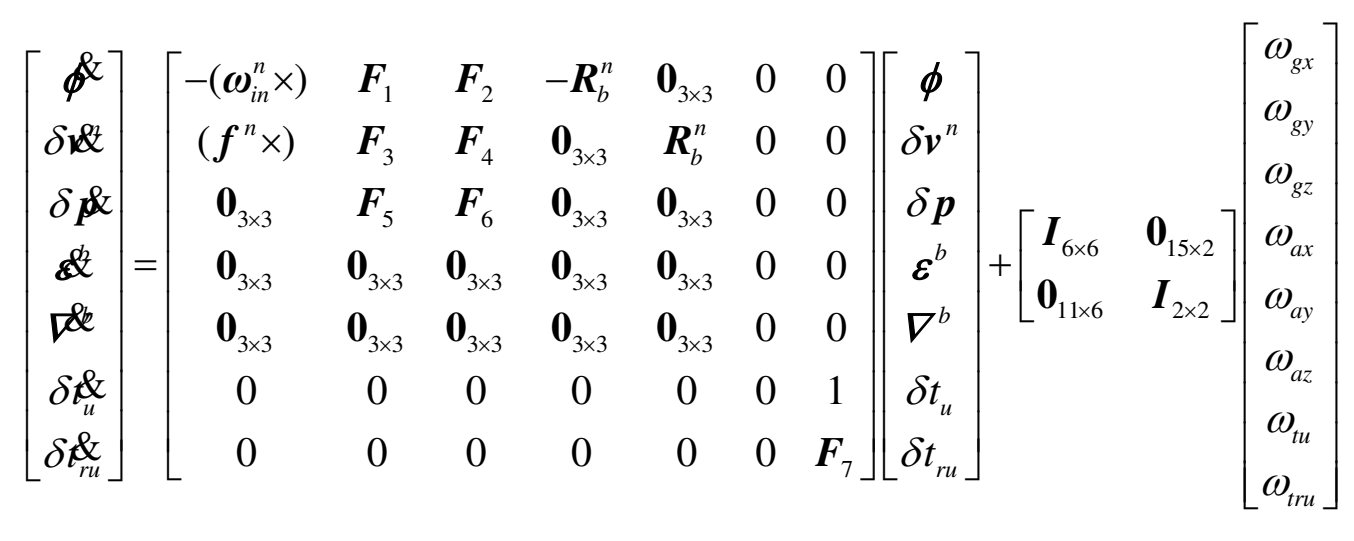


3.4.2. SINS/BDS tightly-coupled measurement equation. The measurement equation of SINS/BDS TCINS is (Gao et al., 2017; Guo et al., 2019)

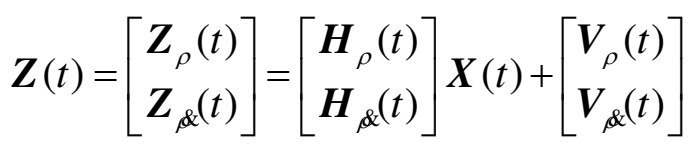

$$
\begin{aligned}
& =\left[\begin{array}{lllll}
\mathbf{0}_{m \times 6} & \boldsymbol{E D}_{a} & \mathbf{0}_{m \times 6} & \boldsymbol{D}_{t u} & \boldsymbol{0}_{m \times 1} \\
\mathbf{0}_{m \times 3} & \boldsymbol{E R}_{n}^{e} & \mathbf{0}_{m \times 3} & \mathbf{0}_{m \times 7} & \boldsymbol{D}_{t u}
\end{array}\right] \boldsymbol{X}(t)+\left[\begin{array}{c}
\boldsymbol{V}_{\rho}(t) \\
\boldsymbol{V}_{\beta<}(t)
\end{array}\right]
\end{aligned}
$$

where $\boldsymbol{E}, \boldsymbol{D}_{t u}$, and $\boldsymbol{V}_{\rho}$ are shown in equation (18), $\boldsymbol{D}_{a}$ is shown in equation (19).

$$
\begin{gathered}
\boldsymbol{E}=\left[\begin{array}{ccc}
e_{1 x} & e_{1 y} & e_{1 z} \\
e_{2 x} & e_{2 y} & e_{2 z} \\
\mathrm{M} & \mathrm{M} & \mathrm{M} \\
e_{m x} & e_{m y} & e_{m z}
\end{array}\right]_{m \times 3}, \quad \boldsymbol{D}_{t u}=\left[\begin{array}{c}
1 \\
1 \\
\mathrm{M} \\
1
\end{array}\right]_{m \times 1}, \quad \boldsymbol{V}_{\rho}=\left[\begin{array}{c}
v_{\rho 1} \\
v_{\rho 2} \\
\mathrm{M} \\
v_{\rho m}
\end{array}\right]_{m \times 1} \\
\boldsymbol{D}_{a}=\left[\begin{array}{ccc}
-\left(R_{N}+h\right) \sin L \cos \lambda & -\left(R_{N}+h\right) \cos L \sin \lambda & \cos L \cos \lambda \\
-\left(R_{N}+h\right) \sin L \sin \lambda & \left(R_{N}+h\right) \cos L \cos \lambda & \cos L \sin \lambda \\
{\left[R_{N}\left(1-e^{2}\right)+h\right] \cos L} & 0 & \sin L
\end{array}\right]
\end{gathered}
$$

3.5. Determination of satellite data validity. In order to ensure the accuracy of the SINS/BDS TCINS, effective satellite observations must be applied. However, the velocity of the near space hypersonic vehicle is over 5 Mach, which may result in abnormal values and observation anomaly in the satellite observations output by the BDS receiver, so as to affect the accuracy of the entire the TCINS. In this paper, the following four methods are applied to perform successive determination on the BDS receiver observations.

(1) According to the receiving time

The time of the BDS receiver output and that of the rising edge of the pulse per second (PPS) is not synchronized. The delay of the BDS receiver used in this paper is within 80 milliseconds. If the time difference is less than the delay time, the package of BDS satellite observation data may be tightly coupled to the navigation; otherwise, the package is considered unusable (Chen et al., 2020b).

(2) According to DOP and satellite elevation angle

The satellite elevation angle is calculated based on the satellite position and the position calculated by the inertial navigation. Satellites with satellite elevation greater than 10 degrees are valid satellites. If the dilution of precision (DOP) is less than 10 and the number of valid satellites is more than 2 , the satellite observations are considered normal. Otherwise, the satellite observations are considered unusable.

(3) According to pseudo-range and pseudo-range rate

The PR and the PRR are calculated from SINS and satellite ephemeris at the current time, which are subtracted by the BDS PR observation and PRR observation at the same time. The validity of satellite data can be determined based on the differences and the thresholds.

(4) According to the incremental of the measurement

The satellite observation data is continuously stored according to the satellite index. If the 
satellite index changes, the satellite observation data within 1 second cannot be used. If the satellite index does not change, calculate the increments of multi-packet PR and PRR that are continuously stored, and compare them with the increment threshold. The validity of satellite observation data can be determined through the incremental changes.

\section{TEST ON SINS/BDS TIGHTLY-COUPLED INTEGRATED NAVIGATION}

4.1. SINS/BDS integrated navigation vehicle field test. A vehicle field test was carried out to verify the SINS/BDS TCINS algorithm. The test was conducted in Xi'an ring expressway, and the track is shown in Figure 5. The initial latitude and longitude of the vehicle were $34.19785^{\circ}$ and $108.82846^{\circ}$, respectively, the initial height was $365.1 \mathrm{~m}$, and the initial velocity was $0 \mathrm{~m} / \mathrm{s}$. A high-precision integrated navigation system was equipped as the reference, called the master INS. The SINS/BDS used in this paper is called the slave INS as shown in Figure 6.

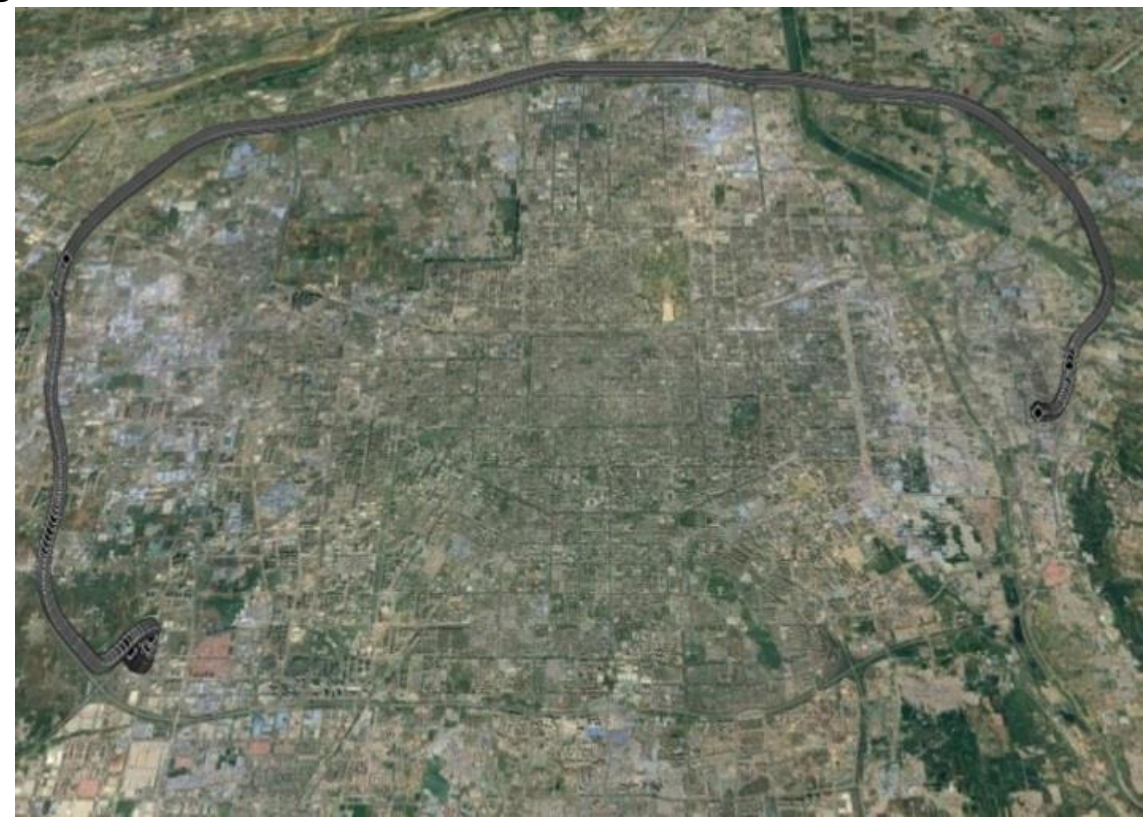

Figure 5. Test track of vehicle in Xi'an ring expressway.
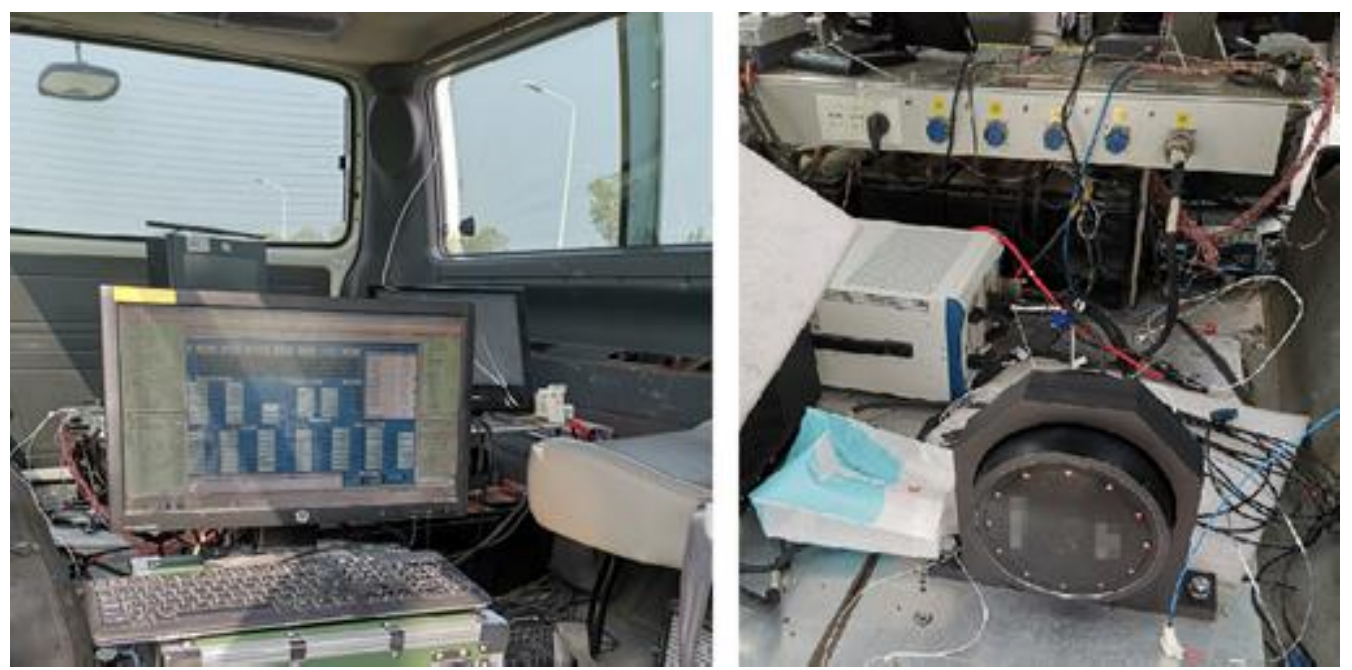

Figure 6. SINS/BDS integrated navigation system and test equipment. 
4.1.1. Comparison of tight coupling and loose coupling. After the collection of the angular increment, velocity increment, and BDS receiver data through vehicle field tests, two combinations of tight coupling and loose coupling are adopted. The comparison results are shown in Figure 7, Figure 8, and Figure 9.

In the vehicle field test, the results indicate that about the TCINS algorithm, the heading angle error is about $0.2^{\circ}$, the pitch angle error is about $0.02^{\circ}$, the roll angle error is approximately $0.03^{\circ}$, velocity errors in the directions of ENU are less than $0.1 \mathrm{~m} / \mathrm{s}$, and the position error is less than $10 \mathrm{~m}$. On the other hand, the loosely-coupled integrated navigation algorithm has the heading angle error of about $0.3^{\circ}$, the pitch angle error of approximately $0.02^{\circ}$, the roll angle error of approximately $0.04^{\circ}$, the velocity errors in the directions of ENU less than $0.15 \mathrm{~m} / \mathrm{s}$, and the position error less than $15 \mathrm{~m}$.
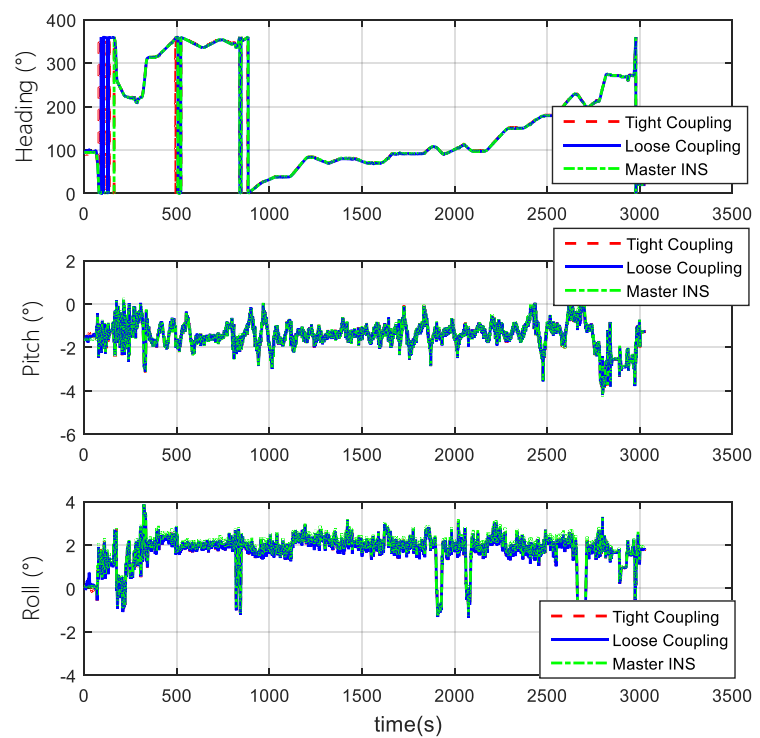

Figure 7. Attitude comparison of tight and loose coupling. 

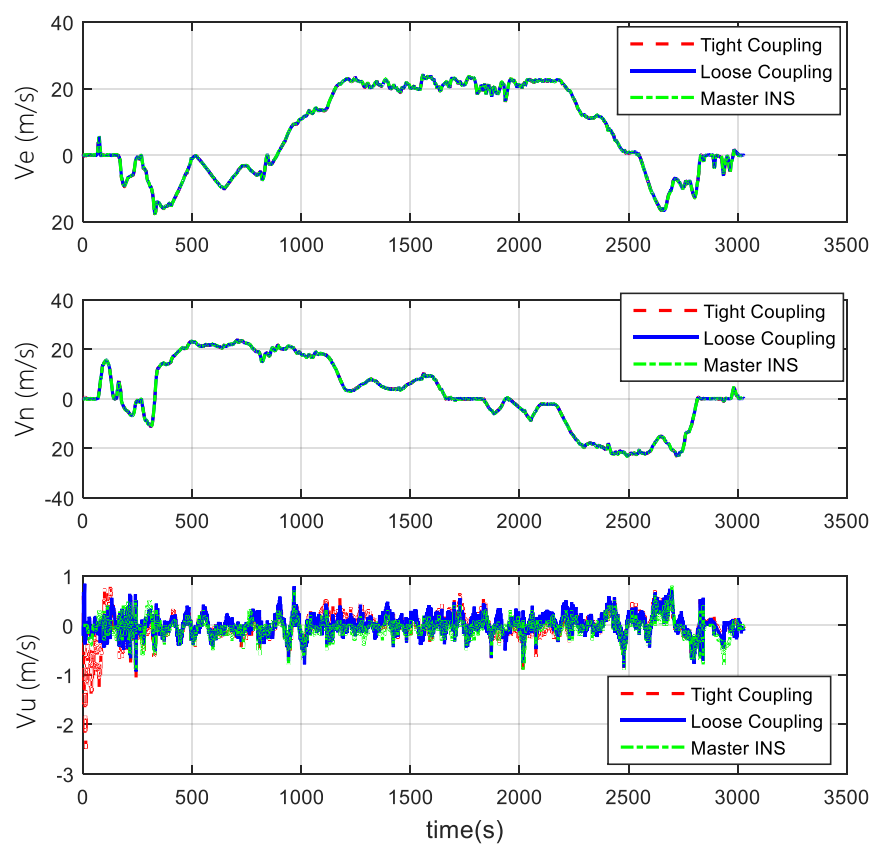

Figure 8. Velocity comparison of tight and loose coupling.
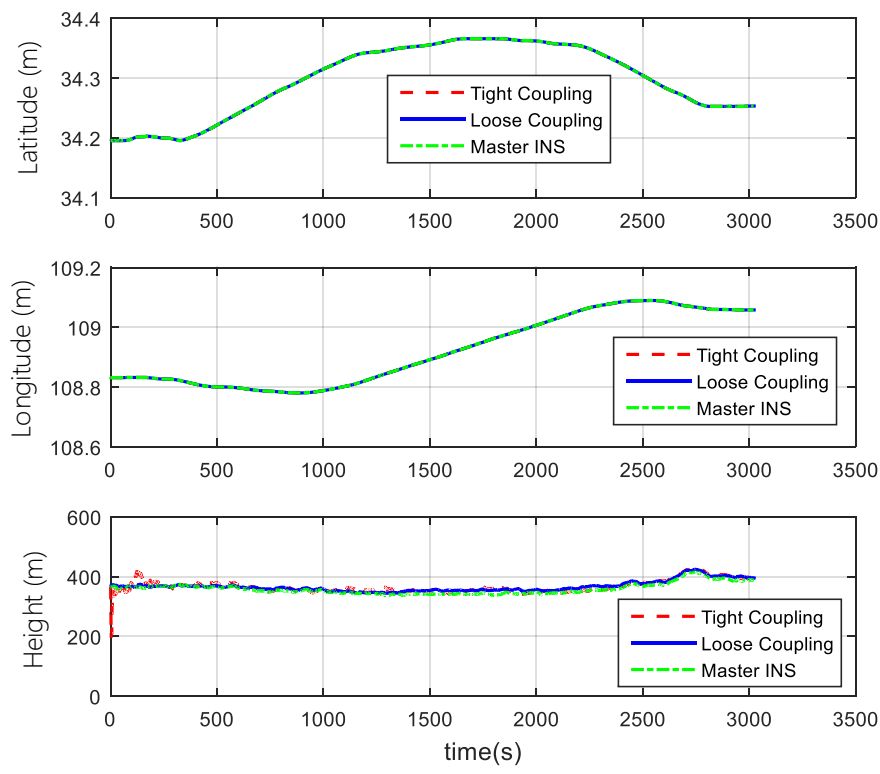

Figure 9. Position comparison of tight and loose coupling.

4.1.2. Comparison of the normal number of satellites and fewer-than-4 satellites. The vehicle field test adopts two cases of normal numbers of satellites and randomly selected three effective satellites to perform TCINS algorithms, respectively. Tight coupling can still perform integrated navigation when the number of effective satellites is fewer than 4 . The comparison of simulation results between the normal number of satellites and the 3 effective satellites are shown in Figure 10, Figure 11, and Figure 12. 

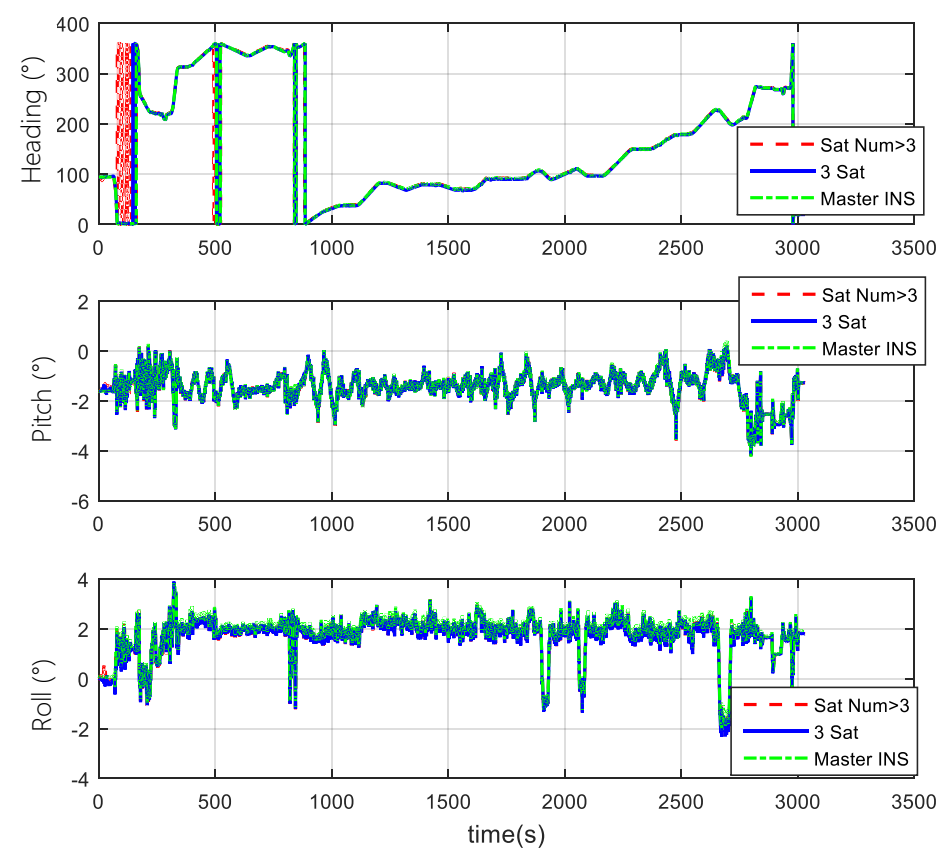

Figure 10. Attitude comparison of navigation by normal number of satellites and 3 sa tellites.
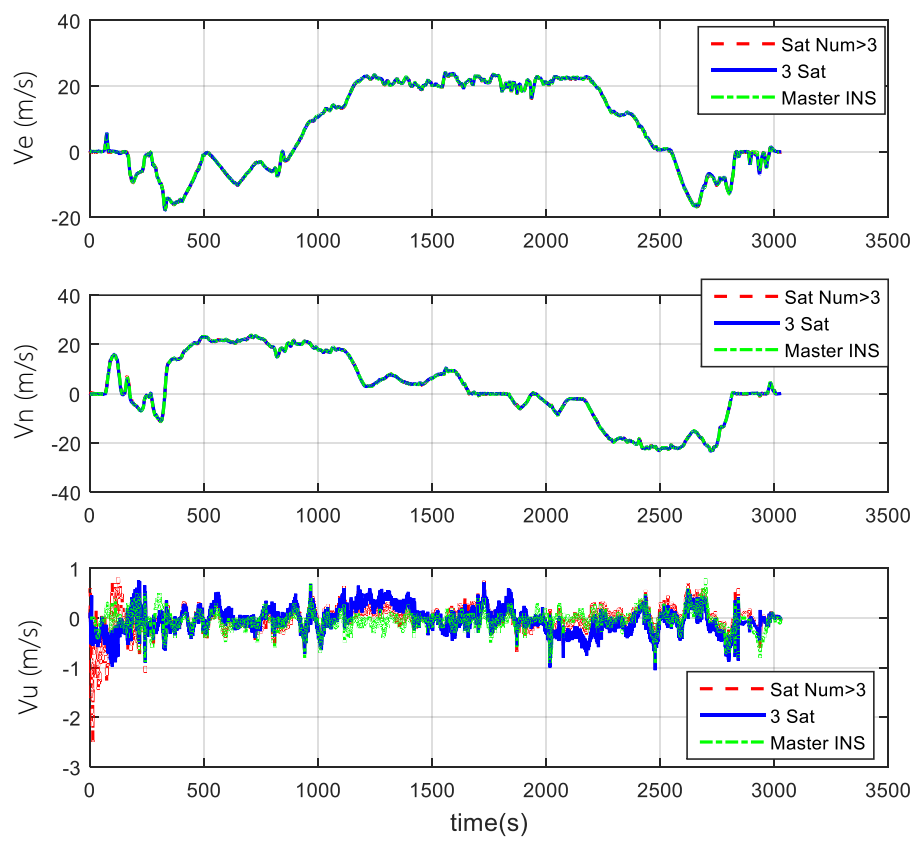

Figure 11. Velocity comparison of navigation by normal number of satellites and 3 sa tellites. 

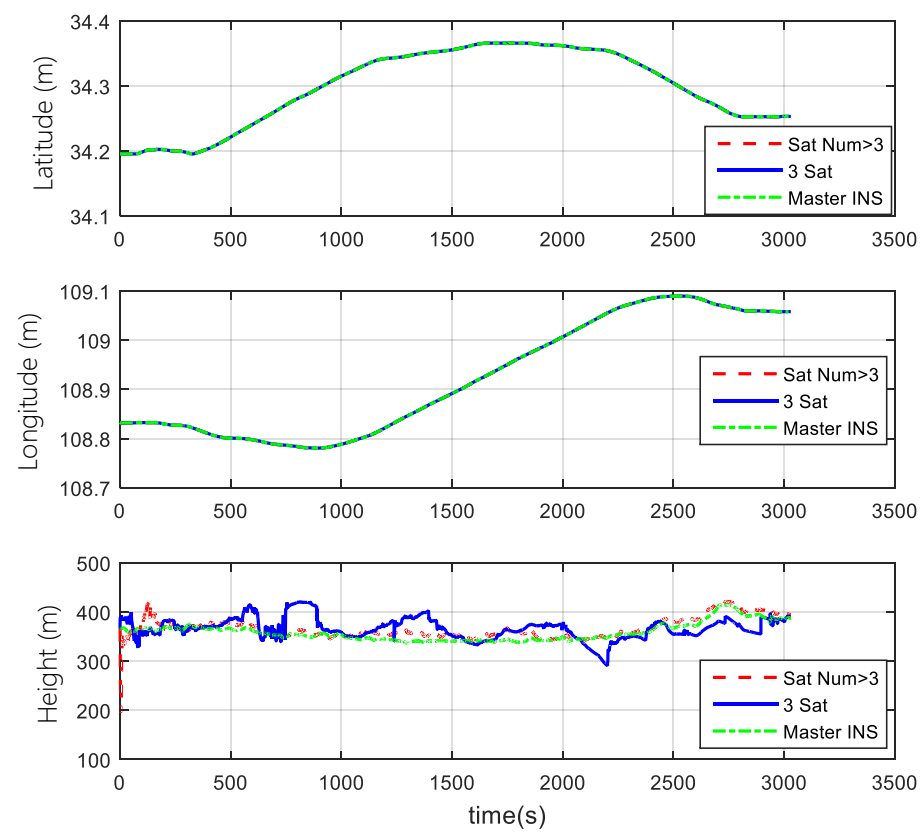

Figure 12. Position comparison of navigation by normal number of satellites and 3 sa tellites

Results demonstrate that, when only 3 effective satellites are used, the maximum heading angle error is approximately $0.5^{\circ}$, the pitch angle error is approximately $0.1^{\circ}$, and the roll angle error is approximately $0.25^{\circ}$. In the case of 3 effective satellites, although the navigation accuracy decreases, it can still perform tightly-coupled integrated navigation normally.

4.2. SINS/BDS integrated navigation test by HWIL simulation. Due to the velocity limitation of field vehicle, the performance of SINS/BDS integrated navigation was verified for hypersonic velocity by the HWIL simulation, as shown in Figure 13. The HWIL simulation tests can truly simulate the flight dynamic environment of hypersonic vehicle, and the navigation algorithm verified by the HWIL simulation tests can meet the flight requirements of hypersonic vehicle. Chen et al. (2020b) has verified the effectiveness of this method. The HWIL simulation equipment includes the real-time simulator, the GNC analysis computer, the 3D display, the three-axis rotation table, the IMU simulator, the BDS simulator, the product interface system, and the actuator load simulator. The on-board equipment includes the on-board computer, the SINS/BDS system, and the actuator system (Chen et al., 2020b). 


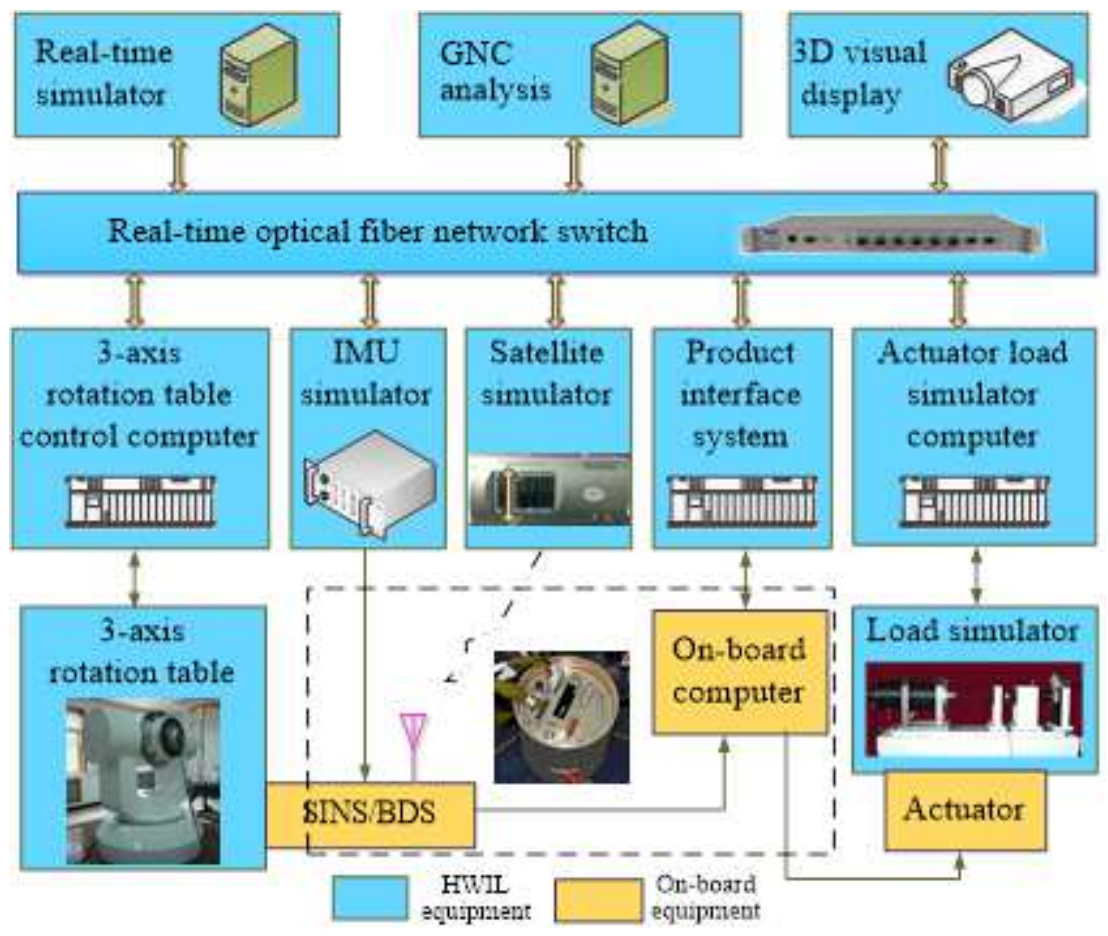

Figure 13. Diagram of HWIL simulation system for hypersonic vehicle SINS/BDS.

4.2.1. Comparison of tight coupling and loose coupling. For the same simulation conditions, the HWIL simulation adopts two combinations of tight coupling and loose coupling, respectively. The results are shown in Figure 14, Figure 15, and Figure 16.

The results of the HWIL simulation test indicate that about the TCINS algorithm, errors of the heading angle, the pitch angle, and the roll angle are approximately $0.1^{\circ}, 0.025^{\circ}$, and $0.03^{\circ}$, respectively. The velocity errors in the direction of ENU are about $0.3 \mathrm{~m} / \mathrm{s}, 0.3 \mathrm{~m} / \mathrm{s}$, and $0.2 \mathrm{~m} / \mathrm{s}$, respectively. The position error is basically within $8 \mathrm{~m}$. On the other hand, in the loosely-coupled integrated navigation algorithm, errors of the heading angle, the pitch angle, and the roll angle are about $0.15^{\circ}, 0.03^{\circ}$, and $0.03^{\circ}$, respectively. The velocity errors in the direction of ENU are about $0.5 \mathrm{~m} / \mathrm{s}, 0.5 \mathrm{~m} / \mathrm{s}$, and $0.32 \mathrm{~m} / \mathrm{s}$, respectively. The position error is basically within $15 \mathrm{~m}$. 

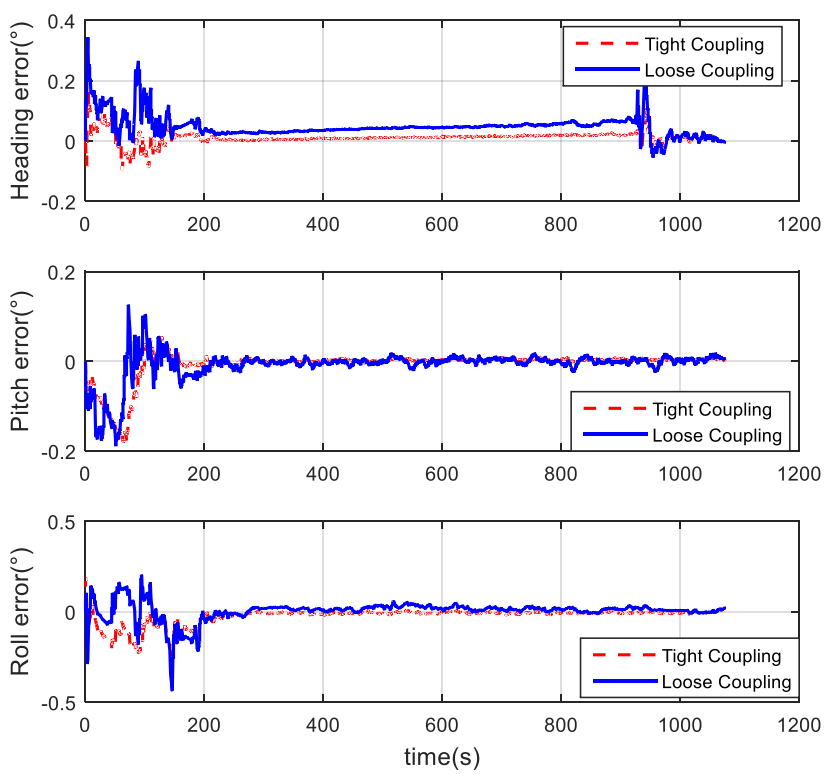

Figure 14. Attitude error comparison of loose and tight coupling.
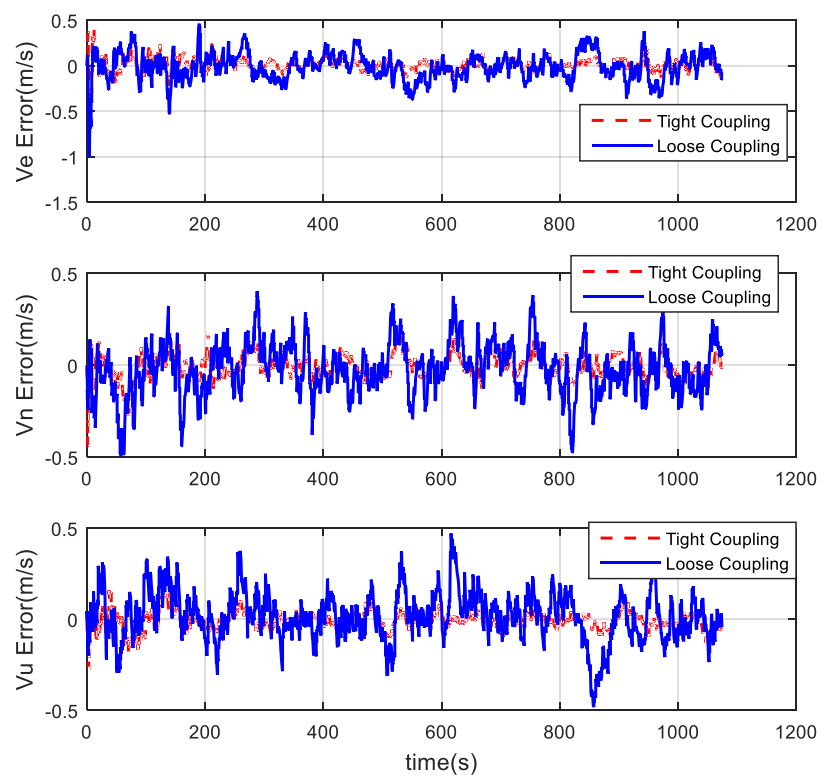

Figure 15. Velocity error comparison of tight and loose coupling. 

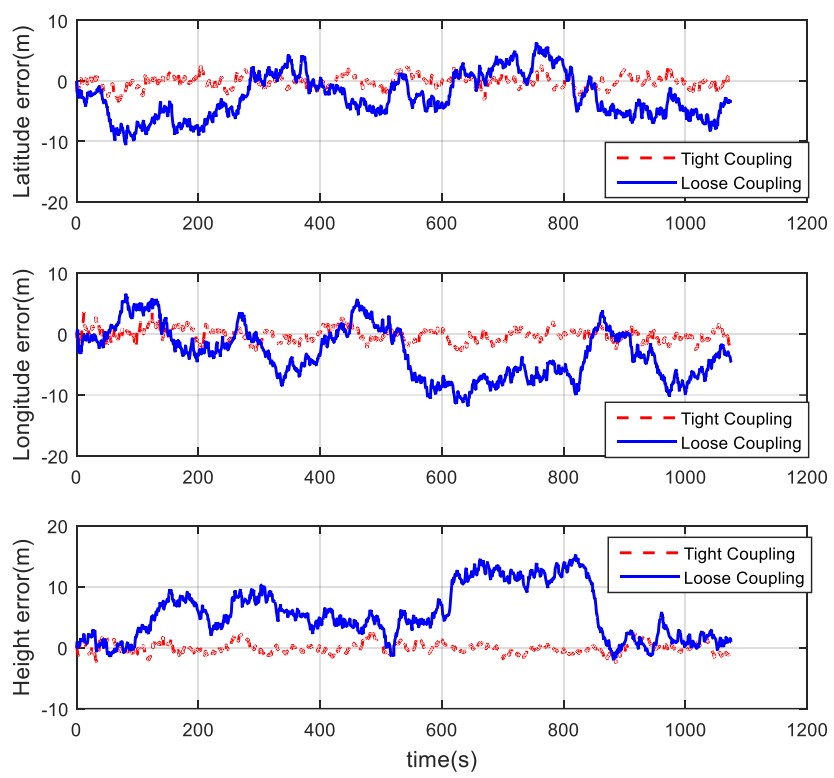

Figure 16. Position error comparison of tight and loose coupling.

4.2.2. Comparison of the normal number of satellites and fewer-than-4 satellites. The HWIL simulation still adopts two cases of normal numbers of satellites and randomly selected three effective satellites to perform TCINS simulations, respectively. The HWIL simulation results are verified that the tight coupling can still perform integrated navigation normally when there are fewer than 4 satellites. The comparison of simulation results between the normal number of satellites and the 3 effective satellites are shown in Figure 17, Figure 18, and Figure 19.

When 3 effective satellites are adopted, the heading angle error is about $0.2^{\circ}$, the pitch angle error is approximately $0.03^{\circ}$, and the roll angle error is approximately $0.04^{\circ}$. The velocity errors in the directions of ENU are about $0.3 \mathrm{~m} / \mathrm{s}, 0.6 \mathrm{~m} / \mathrm{s}$, and $0.8 \mathrm{~m} / \mathrm{s}$, respectively. The maximum horizontal position error and the height error are $180 \mathrm{~m}$ and $200 \mathrm{~m}$, respectively.
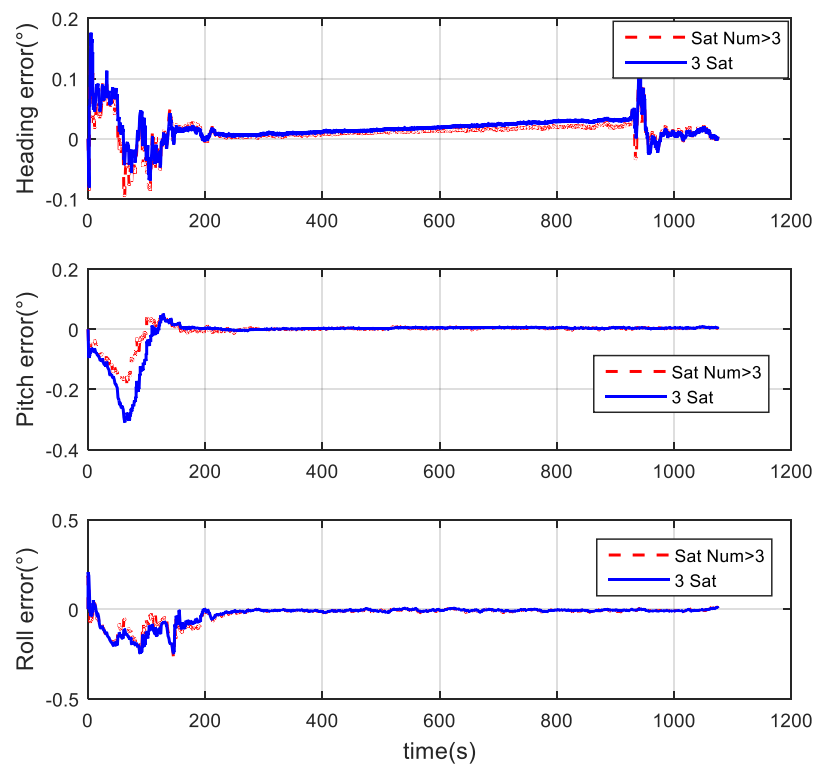

Figure 17. Attitude comparison of navigation by normal number of satellites and 3 sa tellites. 

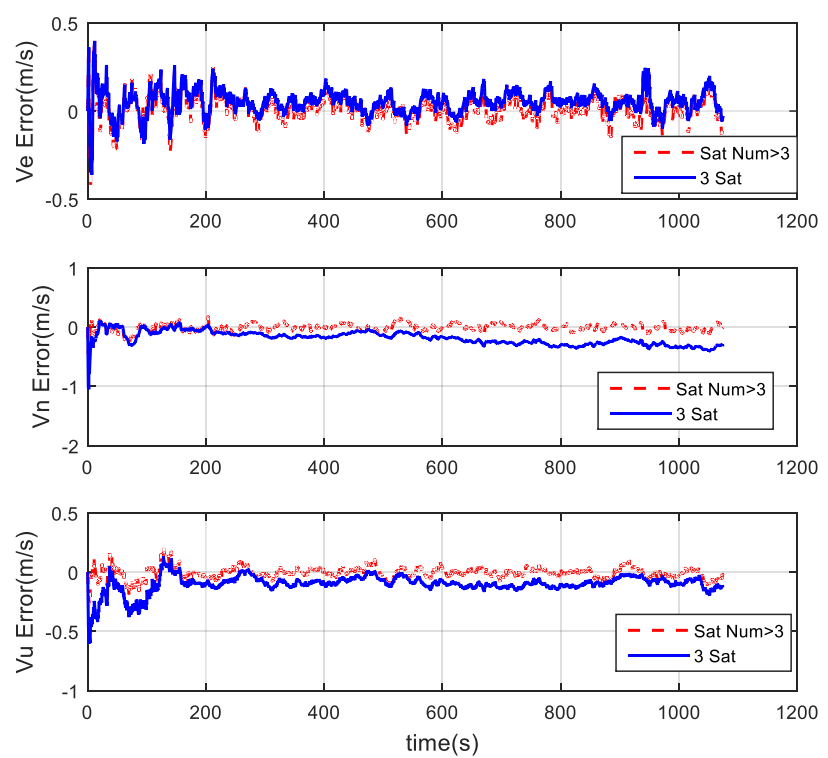

Figure 18. Velocity comparison of navigation by normal number of satellites and 3 sa tellites.
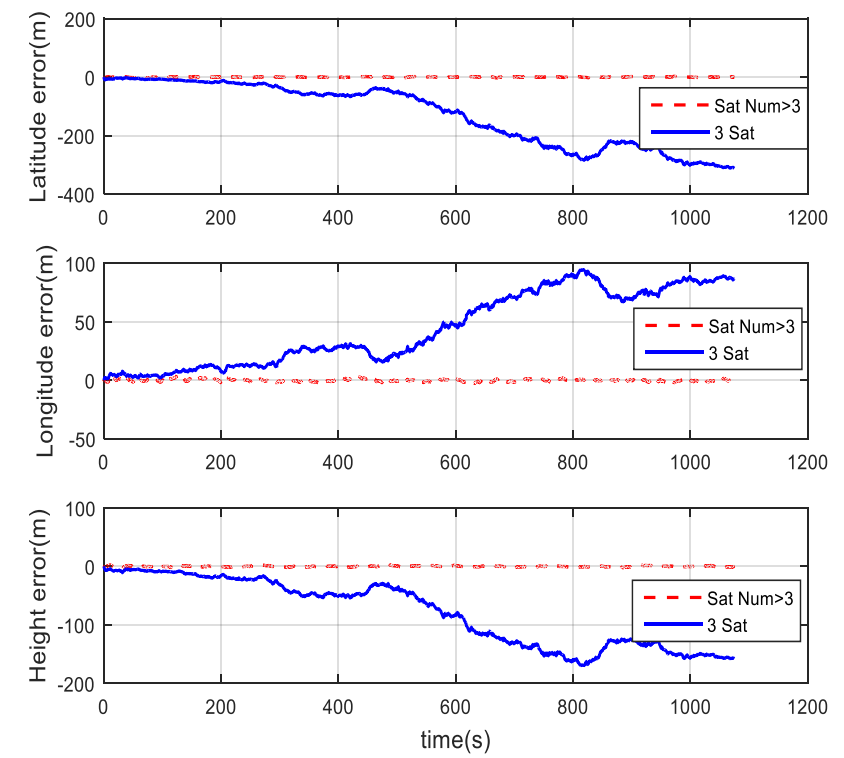

Figure 19. Position comparison of navigation by normal number of satellites and 3 satellites.

5. CONCLUSIONS. In this study, we designed a TCINS algorithm based on the architecture of SINS/BDS integrated navigation system of hypersonic vehicle. Owing to the normal gravity model is no longer applicable in near space, the algorithm uses a J2 gravity model, which is appropriate for near space flight heights over $20 \mathrm{~km}$. We designed the numerical update algorithm of inertial navigation for hypersonic vehicle, and gave the scheme to judge the validity of BDS satellite data. The proposed navigation algorithm was verified by vehicle field tests; hardware-in-the-loop simulation tests are also conducted in order to truly simulate the dynamic environment of hypersonic vehicle flight. Although satellite navigation is 
susceptible to interferences, the tightly-coupled applications can overcome the problem of navigation from fewer than 4 satellites.

\section{Financial support}

This work was supported in part by the Space Science and Technology Innovation Fund of China (2016KC020028) and the Fund of China Space science and Technology (2017-HT-XG).

\section{Conflict of interest}

None.

\section{Author contributions}

K. C. contributed to the conception of the study and performed the experiment;

S. S. P. contributed significantly to analysis and manuscript preparation;

C. Z. Z. performed the data analyses and wrote the manuscript;

G. D. helped perform the analysis with constructive discussions.

\section{References}

Bahm, C., Baumann, E., Martin, J., Bose, D., Beck, R. and Strovers, B. (2005). The X-43A Hyper-X Mach 7 Flight 2 Guidance, Navigation, and Control Overview and Flight Test Results. AIAA/CIRA 13th International Space Planes and Hypersonics Systems and Technologies Conference, Capua, Italy.

Chen, B., Zheng, Y., Cheng, Z., Zhang, H. and Liu, X. (2020a). Review of Astronomical Navigation System for Hypersonic Vehicles in Near Space. Acta Aeronautica et Astronautica Sinica, 1-13. (Chinese)

Chen, K., Shen, F. Q., Sun, H. Y. and Zhou, J. (2019). Hypersonic Vehicle Navigation Algorithm in Launch Centered Earth-fixed Frame. Journal of Astronautics, 40, 1212-1218. (Chinese)

Chen, K., Yin, N. and Liu, M. X. (2018). Equivalency between Two Strapdown Algorithms in Near Space. Aerospace Control, 36, 42-46. (Chinese)

Chen, K., Zhou, J., Shen, F. Q., Sun, H. Y. and Fan, H. (2020b). Hypersonic Boost-glide Vehicle Strapdown Inertial Navigation System/Global Positioning System Algorithm in a Launch-centered Earth-fixed Frame. Aerospace Science and Technology, 98, 105679.

Dai, Q., Sui, L., Wang, L., Zeng, T. and Tian, Y. (2018). An Efficiency Algorithm on Gaussian Mixture UKF for BDS/INS Navigation System. Geodesy and Geodynamics, 9, 169-174.

Duan, M. and Zhou, D. (2020). Finite-time Composite Guidance Law with Input Constraint and Dynamics Compensation. Chinese Journal of Aeronautics, 33, 664-671.

Fan, L., Hu, M. and Jiang, C. (2018). Analytical Long-term Evolution and Perturbation Compensation Mmodels for BeiDou MEO Satellites. Chinese Journal of Aeronautics, 31, 330-338.

Gao, M., Zhao, X., Li, Q. and Wang, Z. (2017). A Method of BDS/SINS Integrated Navigation Fusion based on CKF Algorithm. 2017 13th International Conference on 
Natural Computation, Fuzzy Systems and Knowledge Discovery (ICNC-FSKD), Guilin, China.

Guo, Y., Miao, L. and Xi, Z. (2019). Modeling and Analysis of Measurements of Tightly Coupled GPS/SINS Integrated Navigation System. 2019 Chinese Control Conference (CCC), Guangzhou, China.

Han, H., Wang, J. and Du, M. (2018). GPS/BDS/INS Tightly Coupled Integration Accuracy Improvement Using an Improved Adaptive Interacting Multiple Model with Classified Measurement Update. Chinese Journal of Aeronautics, 31, 556-566.

Hank, J., Murphy, J. and Mutzman, R. (2008). The X-51A Scramjet Engine Flight Demonstration Program. 15th AIAA International Space Planes and Hypersonic Systems and Technologies Conference, Dayton, Ohio.

Ma, Z., Zhou, Z. H., Wang, P., Hu, J. B. and Ke, B. Q. (2019). Analysis of Airworthiness Requirements of BDS Airborne Equipment for Aircraft Tracking Only. Acta Aeronautica et Astronautica Sinica, 40, 188-198. (Chinese)

Niu, Q., Yuan, Z., Chen, B. and Dong, S. (2019). Infrared Radiation Characteristics of a Hypersonic Vehicle under Time-varying Angles of Attack. Chinese Journal of Aeronautics, 32, 861-74.

Savage, P. G. (1998a). Strapdown Inertial Navigation Integration Algorithm Design Part 1: Attitude Algorithms. Journal of Guidance, Control, and Dynamics, 21, 19-28.

Savage, P. G. (1998b) Strapdown Inertial Navigation Integration Algorithm Design Part 2: Velocity and Position Algorithms. Journal of Guidance, Control, and Dynamics, 21, 208-221.

Schmidt, G. T. (2015). Navigation Sensors and Systems in GNSS Degraded and Denied Environments. Chinese Journal of Aeronautics, 28, 1-10.

Steffes, S. (2012). Real-time Navigation Algorithm for the SHEFEX2 Hybrid Navigation System Experiment. AIAA Guidance, Navigation, and Control Conference, Minneapolis, Minnesota.

Walker, S., Sherk, J., Shell, D., Schena, R., Bergmann, J. and Gladbach, J. (2008). The DARPA/AF Falcon Program: The Hypersonic Technology Vehicle\# 2 (HTV-2) Flight Demonstration Phase. 15th AIAA International Space Planes and Hypersonic Systems and Technologies Conference, Dayton, Ohio. 